\title{
A glance at the history of uniportal video-assisted thoracic surgery
}

\author{
Tommaso Claudio Mineo ${ }^{1}$, Vincenzo Ambrogi ${ }^{1,2}$ \\ ${ }^{1}$ Department of Surgery and Experimental Medicine, ${ }^{2}$ Thoracic Surgery, Official Group of Awake Thoracic Surgery Research, Policlinico Tor \\ Vergata University, Rome, Italy \\ Contributions: (I) Conception and design: TC Mineo; (II) Administrative support: V Ambrogi; (III) Provision of study materials or patients: V \\ Ambrogi; (IV) Collection and assembly of data: V Ambrogi; (V) Data analysis and interpretation: All authors; (VI) Manuscript writing: All authors; \\ (VII) Final approval of manuscript: All authors. \\ Correspondence to: Tommaso Claudio Mineo. Tor Vergata University, Rome, Italy. Email: mineo@uniroma2.it.
}

\begin{abstract}
In the history of thoracic surgery, the advent of video-assisted thoracic surgery (VATS) had on effect equivalent to that provoked by a true revolution. VATS successfully allowed minor, major and complex procedures for various lung and mediastinal pathologies with small incision instead of the traditional accesses. These small incisions abolished ugly scars, generated less acute and chronic pain, reduced hospital stay and costs, allowed faster return to normal day life activities. Conventional VATS was initially performed through 3-4 ports and rapidly evolved to uniportal or single portal access [uniportal video-assisted thoracic surgery (uniVATS)]. First uniportal procedures were published in 2000. In 2010, uniportal technique for lobectomy was described. Focused experimental courses, live surgery events, the internet media favored the rapid diffusion of this technique over the world. Major and complex uniVATS lung resections involving segmentectomy, pneumonectomy, bronchoplasty and vascular reconstruction, redo VATS, en bloc chest wall resections have been accomplished with satisfactory outcomes. Interestingly, different uniportal approaches and techniques are emerging from a number of VATS centers particularly experienced in the mini-invasive thoracic surgery. As confidence grew, in 2014, the first uniVATS left upper lobectomy via the subxiphoid approach was reported. This novel technique is quite challenging but appropriate patient selection as well as availability of dedicated instruments allowed to perform procedures safely. The diffusion of uniVATS paralleled with the development of nonintubated awake anesthesia technique. In 2007 the first nonintubated lobectomy was described. In 2014 the first single port VATS lobectomy in a nonintubated patient with lung cancer of the right middle lobe was accomplished. The nonintubated uniVATS represents an intriguing technique, so that very experienced thoracoscopic surgeons may enroll to surgery elderly and high risk patients. Decreased postoperative pain and hospitalization, faster access to the radio-chemotherapy and diminished inflammatory response are important benefits of the modern approach to the thoracic pathologies. The history of uniVATS documented a constant and irresistible progress. This technique may further provide unthinkable surprises in next future.
\end{abstract}

Keywords: History; thoracic surgery; video-assisted thoracic surgery (VATS); uniportal VATS (uniVATS)

Received: 05 April 2017; Accepted: 03 April 2017; Published: 07 November 2017.

doi: $10.21037 /$ jovs.2017.10.11

View this article at: http://dx.doi.org/10.21037/jovs.2017.10.11

\section{Introduction}

We are sure of not exaggerating if we affirm that singleport video-assisted thoracic surgery (VATS) or uniportal video-assisted thoracic surgery (uniVATS) is the "talk of the town" in thoracic surgery, and it will be so for a long time. It surely represents the new frontier of minimally invasive surgery, with an increasing diffusion and consolidation in many centers all over the world. It attracts the interest of 
both the newest generations of thoracic surgeons but also of chest physicians, who are keener to consider surgical treatments for their patients. Furthermore, the patients themselves are more incline to accept the chest procedure, once aware about the self-evident advantages of this modern surgical approach.

It is evident that only very few topics in thoracic surgery, among those debated for many decades, aroused such an interest and raised such an enthusiasm as uniVATS. Although this impression is not quantifiable, we feel that the present interest for uniVATS is even more evident and widespread than the one about multiport VATS in the early 1990s.

Conventional VATS undoubtedly represented the watershed with the traditional open thoracic surgery. uniVATS particularly, results from both important technological developments and acquired surgical skills, that trigger the natural challenging attitude of the surgeons, especially the young ones, who are more receptive to innovative solutions.

In order to better understand the features of this new surgical technique we have to start from multiport VATS. In 1992, VATS stormed into the world of thoracic surgery after a long age of wide surgical accesses and traditional surgical technique (1-5). These operations had many positive results that obliged also to accept their unwarranted complications and sequelae. New endoscopic instruments, in primis variable angulated endostaplers, and new endoscopic cameras with a panoramic view of the thoracic cavity, made possible the beginning of a new era with the refusal of the no longer acceptable flaws of the traditional surgical approaches.

Reduced surgical trauma with lesser acute and chronic pain, shorter hospital stay, decreased morbidity, safeguard of the harmonic cosmesis of the chest wall and quicker return to the domestic and occupational activities are the most relevant achievements featured by the numerous VATS operations. Accordingly, VATS also modified patient's awareness, as they used to be reluctant and often contrary to surgical treatment. With the advent of multiport VATS, they released more promptly their consent to a procedure that allowed smaller surgical scars and lesser postoperative discomfort with similar therapeutic outcomes.

Multiport VATS was employed in all fields of thoracic surgery, from lung to esophageal and mediastinal surgery with lesser morbidity and faster recovery when compared to classic open thoracic access. During a 20 -year timespan the technique has gradually grown, thus allowing the fulfillments of many old desires and expectations of the surgeons.

It was obvious from the very beginning that the first step would have been the progressive reduction of the number of skin mini-incisions. Indeed, starting from the initial three-four ports, the accesses were reduced to two and, shortly after, to one, $2-6 \mathrm{~cm}$ length, that equally allowed similar safe procedures by means of further evolved instruments.

Having said that, we have to address a glance to the history of uniVATS. It looks short in time, but very significant and characterized by the continuous progress through challenging phases. This pathway started from minor procedures and lead gradually towards the major and the most complex ones, that at first one could had figured feasible only in open surgery or, alternatively, in very experienced hands via multiport VATS.

\section{The start}

We can identify an old and a recent stem in the history of uniVATS. The first one sprung from Jacobaeus (6) who in 1910, after having positioned a light in a common cystoscope, created the first thoracoscope. Therefore, Hans Christian Jacobaeus can be considered the first physician to employ the uniportal technique to enter the pleural cavity, perform biopsies and divide pleural adhesions. This event represents the commonly-accepted origin of thoracoscopy or pleuroscopy that was usually performed in spontaneously breathing patients using local anesthesia $(7,8)$. This technique was unquestionably useful to investigate the normal and pathological aspects of the pleural cavity and the lung. The original "Jacobaeus operation" was performed through this unique skin incision, whose exact length remains unknown. Two incisions were occasionally used to improve visualization $(9,10)$. For many decades this procedure was used by pulmonologists for mainly diagnostic purposes (11). Following these experiences, the operation was furthermore popularized and indications extended to treatment of pneumothorax, pleural effusion with talc pleurodesis and sympathectomy (11).

These procedures, still more diagnostic than therapeutic, had quite a satisfactory diffusion but they missed a significant success and, until the 70's, the role of thoracoscopy remained predominantly confined to diagnose and treat pleural diseases $(10,12)$. In the following years, the advent of antibiotics, and above all, the lack of innovative technologies favored its progressive withdrawal. 


\section{The early evolution}

The modern stem of uniVATS has a relatively short but very intense story, with a series of increasing surgical achievements that emphasized its safety and efficacy both in minor and major operations (13). uniVATS major pulmonary resections follow the oncological principles of open surgery allowing anatomical dissection of the hilar structures, as well as complete radical lymphadenectomy for non-small cell lung cancer. Despite its relatively short history, the diffusion of the technique has been instead quick and steady. This is very uncommon and surprising, especially compared to other techniques of open thoracic surgery or with the more recent conventional VATS.

Thus, one could wonder whether such a fast diffusion (14-18) has been triggered by the rapid development of technology, by the modern mass media, or by faster learning abilities of the youngest surgeons. Nevertheless, must give credit to those prominent personalities in the field of minimally-invasive thoracic surgery that in recent years were so deeply involved in teaching and popularizing the new procedure, remarkably accelerating the learning curves (19-21).

We have a unique affirmative answer to all these questions. Indeed, surgery should always be considered a continuously evolving art. Nowadays, the technological evolution and the quick fruition of the novel surgical progresses walk hand in hand and find a formidable ally in the speed of communication. It helps to dissolve doubts and uncertainties thus more easily determining their success.

Obviously, the fortune of uniVATS is built on the wellknown prerogatives of multiport VATS, which represents the true technological innovation in chest surgery.

Since history needs dates, we want to list some of those in order to better understand the growth of this relatively young field of thoracic surgery. Initially, it was restricted to minor operations, but nowadays it extends to major anatomic resections and reconstructions. This rapid development forced many surgeons to affirm that we are in the uniVATS age, whose boundaries are still unsettled.

We state that in 1998 Yamamoto et al. (22) used a single $2 \mathrm{~cm}$ skin incision at the midaxillary line level to perform successful lung resection, albeit limited to 6 patients with pneumothorax.

In literature one can find that in 2000, Nesher et al. (23) from Haifa described with simplicity a thoracic sympathectomy for palmar hyperhidrosis accomplished through an unclear thoracoscopic VATS access that cannot be defined a uniportal approach according to the modern and accepted definition of 2-5 cm of length.

In 2000, at the Thoracic Surgery and Interdisciplinary Symposium on the Threshold of the Third Millennium held in Naples, Migliore et al. (24) presented a series of 58 different procedures performed through a single port. In this occasion these authors described a handcrafted $20 \mathrm{~mm}$ flexible circular trocar, rigid enough though to avoid its collapse when introduced into chest cavity (25). Several pathologies including large anterior mediastinal masses, staging of lung cancer, pleural effusions sympathectomy, stage II empyema and pneumothorax were approached by Migliore through this technique, that he strongly advocated $(26,27)$. This short communication is enough to affirm that Migliore should take credit for having first endeavored uniportal thoracic surgery in different pathologies, having anticipated the enormous potentialities of the unique access to the pleural cavity. Subsequently, the same authors (28) extended the indications to uniVATS and confirmed what they had already described attributing to the single-trocar technique the importance and the role that everybody now recognize to uniVATS procedures.

Soon after, in 2004 Rocco et al. (29) published their initial experience with uniVATS for mediastinal biopsies and wedge pulmonary resections for both diagnosis of interstitial lung diseases and treatment of primary spontaneous pneumothorax (30,31). In 2005, Jutley et al. (32) reported a comparison between the standard threeport VATS and uniVATS for surgical management of spontaneous pneumothorax and the latter demonstrated safer and more effective.

We should acknowledge to Migliore first and Rocco after, the merit of having introduced uniVATS and intuited its enormous benefits. Furthermore, they established the principles of this technique that is based, such as conventional VATS, on the proficiency in open surgery and its variations. Obviously, the early experience with uniVATS technique is also based on the development of new optic and endoscopic articulating instruments. Safety, feasibility, reduced pain and paresthesia, better cosmesis are soon highlighted as the main advantages described by the proponents of this engaging technique.

Minor operations such as wedge resections, pericardial window, sympathectomy, evacuation of hemothorax, treatment of primary and secondary pneumothorax, mediastinal biopsies, were the most common procedures in the initial period $(19,33)$. However, the experience gained by various dedicated centers, both in Europe and in Asia, 
lead to the endeavor of major operations as well. This last challenge produced another further and important jump forward in the evolution of minimally invasive surgery. In expert hands the uniVATS technique allowed the execution of the most complex cases in the same manner as with triple or double port access. More challenging operations such as completion pneumonectomies, sleeve lobectomies, pulmonary resection after high doses of chemotherapy, vascular reconstructions are now successfully performed in advanced and selected centers. Since then, in quick and amazing succession, uniVATS very demolitive and reconstructive lung operations were reported by Diego Gonzalez-Rivas and his group (34-46).

\section{The recent progress}

In order to list the dates as a true story requires, a crucial moment is June 2010 when the first uniVATS lobectomy was carried out by Gonzalez-Rivas at La Coruña Hospital (34). From that time, the second and most fruitful period of the uniportal technique has started, directly born from the multiport VATS. Initially, the technique was only used to perform lower lobectomies, but its rapid improvement favored the extension of the procedure to upper lobectomies (20), segmentectomy (35) and pneumonectomy (36). The fast development of the uniVATS technique enlarged the indications and reduced contraindications. The success of all these procedures depends on both surgeon's skills and availability of specific VATS instruments, which can be similarly used in individuals with various body size. In this regard, it is of prominent importance to mention the role of endostaplers, specifically designed to improve their efficacy and safety, when used on the hilar structures. Surgeons nowadays should be particularly well acquainted with the use of endostaplers, knowing every technical detail in order to select case-by-case the most appropriate model.

Controversies emerged about the employ of uniVATS in both advanced stages of lung cancer and mediastinal lymphadenectomy that is essential in lung cancer surgery. One should consider that there is an increased risk of major bleeding and technical difficulties when performing radical resection in advanced stage. Therefore, a correct preoperative planning of the operation and a careful dissection of the vascular structures decrease the risk of complications during uniVATS. The surgeon should acquire an acute perception about the need of conversion to thoracotomy which should never be considered as a failure of the initial approach.
The surgical progress achieved in uniVATS is unquestionably merit of the group lead by Gonzalez Rivas and the dates signaled this irresistible march. In 2012, they described the first lobectomy with chest wall resection by posterior incision (37). In 2013, the first bronchial sleeve lobectomy (38) and the first lobectomy with pulmonary artery reconstruction (39) were carried out. In 2014, they reported the first bronchovascular right upper lobe reconstruction (42) and the first double sleeve lobectomy for non-small lung cancer $(43,44)$. In 2015 , the same group accomplished a lobectomy with en-bloc chest wall resection (45) and right upper sleeve lobectomy (13). Finally, in 2016, the first carenal sleeve resections were performed and published (46).

It is still an open debate whether an equal systematic nodal dissection can be performed via VATS or thoracotomy approach (47). Delgado et al. (48) reported better results in uniVATS than in multiport VATS for mediastinal lymphadenectomy. More recently, Liu et al. (49) observed that the quantity and quality of dissected lymph nodes was similar, whatever the used VATS approach.

On the other hand, in 2015, Mu et al. (50) demonstrated an inferior overall number of dissected lymph nodes and nodal stations sampled in uniVATS lobectomy. In 2016, Shen et al. (51) showed that duration of lymphadenectomy was longer in uniVATS while duration of lobectomy was shorter. Obviously, all these data are related to the learning curve of each surgeon (52). In this regard, in 2011 Gonzalez-Rivas et al. (53) described their initial experience in VATS lobectomy comparing three different periods of time: the beginning [2007-2008], the intermediate [2008-2009] and the last [2009-2010]. The analysis of these different periods showed that the learning curve was crucial for improving in this technique. The last period resulted in lower operative time than the previous ones. In the following years, benefits and drawbacks of uniVATS compared with multiport VATS and thoracotomy were investigated, with several authors focusing on postoperative outcomes.

Compared to thoracotomy VATS has been associated with decreased mortality (54) and morbidity including major cardiopulmonary complications, atelectasis, wound infections and atrial fibrillation (55), better pain and quality of life (56). The shorter hospital stay is the most important feature, so that VATS is now considered the best approach for elderly patients (57), or with multiple comorbidities (58).

Conversely, there are discordant opinions between the postoperative outcomes of uniVATS and multiport VATS $(59,60)$. In 2016, Harris et al. (61) reviewed eight large 
retrospective studies showing that uniVATS was associated with lower morbidity, shorter duration of chest tube stay and no significant differences in number of dissected nodes, conversions to open thoracotomy or operative time.

Reduction of intraoperative blood loss and postoperative pain with a higher patient's satisfaction score in uniVATS emerged from a propensity matched comparative analysis by Dai et al. (62). Similarly, in 2016, French et al. found no significant difference in conversion rate, perioperative bleeding, mortality rate and median operative time between Uni and multiport VATS (63). From a retrospective comparison of Uni Vs multi VATS lobectomies by Chang et al. (64) no differences in operative time, postoperative 30-day mortality, chest tube permanence, number of removed lymph nodes, hospital stay and reoperation rate emerged.

It has been suggested that immune response is better preserved after VATS compared to thoracotomy (65-67). Our recent studies showed that the same effects of lower immunological suppression and reduced inflammatory response can be detected when comparing uniVATS and multiport VATS (68). As a consequence, given the lower trauma of uniVATS, it is hypothesizable that this technique could favor an improved long-term survival and impact on tumor recurrence in patients with primary or secondary lung cancers (69).

On the basis of this overwhelming literature why would one rather uniVATS? First, the personal preference of the surgeon, deriving from a consolidated experience in conventional VATS. Second, a number of advantages over the multiport VATS including a single, minimal and more cosmetic skin incision, less postoperative pain and discomfort, shorter hospital stay often limited to one day in expert hands, more rapid access to radio or chemotherapy programs. Most important point, the faster patient's acceptance of the thoracic operation, that was often difficult to obtain especially when complex procedures were prospected.

And yet, as in all surgical procedures, all that glitters are not gold. Indeed, uniVATS entails important risks that have to be considered. Limited surgeon's experience, inappropriate patient's evaluation associated with uncontrolled bleeding and technical difficulties can be reasons of failure or conversion (70).

Location of the $2.5-5 \mathrm{~cm}$ unique skin incision made according to muscle sparing technique is of paramount importance for the safety and the good outcome of the operation $(71,72)$. Generally, a single incision located between the fourth and the sixth intercostal spaces along the mid-axillary line can be used in the majority of cases even for very complex lung resections, respecting the established oncological principles. However, the incision site is very variable and may change according to the surgeon's preference as well as learning curve.

Different uniportal access and technique are emerging from many VATS centers in the world. Notably, uniVATS have been performed through transcervical, trans-subcostal, transaxillary, transsternal, transdiaphragmatic and more recently subxiphoid approaches. The subxiphoid single port approach represents the ultimate achievement of the unstoppable evolution of VATS.

\section{A bold access}

The 2014 is another cornerstone year in the intense history of uniVATS. After a large previous experience on transthoracic uniVATS, Liu et al. from Taiwan (73) published the first successful uniVATS left upper lobectomy via the subxiphoid approach. The group of Liu chose the subxiphoid access in order to avoid unpleasant thoracic scars, especially in young females, and to reduce postoperative chest pain. This approach helped to avoid the limitation caused by narrow rib spaces and reduced the risk of intercostal nerve injury. Technically, this incision provided better angles for hilar dissection and for insertion multiarticulated staplers. In 2016, the same group reported 39 patients who underwent surgery via a $3-4 \mathrm{~cm}$ vertical subxiphoid single incision (74). The technique through this route is similar to that used during conventional single-port access. The authors strongly noted that a consolidated single port experience is fundamental to accomplish all steps of the procedure without instrumental fighting. Appropriate patient selections and particularly dedicated instruments are necessary to perform a safe operation. Furthermore, they suggested that anatomical resection of every lobe and traditional segmentectomy can be safely accomplished with this single incision approach. On the other hand, limitation exists for radical mediastinal lymphadenectomy as well as for control of major bleeding. One should acknowledge the merits of this approach for decreased postoperative pain, well accepted better cosmesis and easier specimen retrieval in comparison with transthoracic route.

In recent years, the subxiphoid approach was adopted for VATS extended thymectomy in non-thymomatous myasthenic patients, in selected thymomas and in small sized anterior mediastinal tumors (75). The subxiphoid 
approach was first used Kido et al. (76) in 1999. This engaging approach proved that extended thymectomy is technically feasible and safe. Benefits of this unique incision over the lateral transthoracic access includes a better operative view of the cervical area (77) and of bilateral pleural cavities (78) and a relatively less pain. Another clear and described merit is a well accepted impact on cosmesis. In recent period, we experienced this approach in nonthymomatous and thymomatous patients in awake modality.

Chen et al. (79) noted that the subxiphoid access also allows partial pericardial and wedge lung resection when necessary. This approach is very attractive, but due to its different surgical view and operative technique, it requires sufficient training in both transsternal and multiport VATS thymectomies and in the use of new instruments.

\section{The awake anesthesia in progress}

Awake-nonintubated minimally invasive thoracic surgery rightly occupies a significant space in the history of uniVATS. We know that awake-nonintubated minor thoracic operations have been performed since the very first steps of thoracic surgery. However, this modality ultimately failed to gain acceptance for several reasons, so that interest progressively vanished for the lack of a diffuse consensus.

All advantages of general anesthesia and double lumen intubation are well known. Nevertheless, the adverse effects including an increased risk of pneumonia, neuromuscular problems, risk of major airways injuries and composite spectrum of lung lesions (barotrauma, volotrauma, biotrauma and atelectrauma) are sufficiently recognized $(80,81)$.

Double-lumen intubation itself can cause important ventilation-related injuries in approximately $4 \%$ of major lung resections, with a mortality rate as high as $25 \%$ (82). Residual neuromuscular blockade, postoperative nausea and vomit, inflammatory response $(83,84)$ during lung operations incidentally associated to postoperative complications, encouraged the research of new methods of anesthesia that could overcome these potentially dangerous side-effects.

Over the last decade, surgical advances such as multiport VATS and uniVATS allowed surgeons to consider new anesthetic methods including non-general anesthesia and non-invasive monitoring as well $(85,86)$. Nonintubated anesthetic technique for thoracic operations reinforced the virtues and benefits of VATS procedure $(84,87)$. Thus, there is a growing interest in nonintubated modality during which thoracic surgery is carried out in awake and spontaneously ventilating patients, with minimal sedation and local regional anesthesia or under general anesthesia with supraglottic airways devices (88).

Thoracoscopic minor or major operations in awake and collaborative subject is a new approach, to be considered an important achievement which is gaining progressive consensus especially amongst the most advanced multi and uniVATS centers (89-92).

Of course, the development and the success of this approach depend on the training and the commitment of each member of the surgical team. A prominent role is also played by the anesthesiologists, who needs to be well trained on nonintubated technique and should act in the operatory room as an integrated component of the surgical team $(80,88,93,94)$. Rapid and straightforward intubation of a patient lying on lateral or supine decubitus, whenever required by an arising complication, should always be part of the background $(80,88,94)$. According to this perspective, all the instruments and medications required to perform a fast-track intubation should be immediately available.

Complications can be anticipated and prevented by an appropriate conversion to intubated general anesthesia $(90,94)$. Therefore, sudden and unforeseen technical difficulties, uncontrollable bleeding, negative influence of mediastinal and diaphragmatic movements should be promptly signaled.

Risk-evaluation is anyway pivotal, and relevant differences in terms of risks between the minor and major procedures in the awake patients exist. The risks in a minor operation such as wedge resection is significantly lower than in an anatomic lung resection, where an uncontrolled bleeding can create serious problems. This is the main reason why the nonintubated major lung resections must be accomplished only by very experienced thoracoscopic surgeons. Once again, we noted that the experience is the only way to reduce the rate of complications and conversions when working in a minimally invasive setting. Generally, it is wise to start with minor operations and, once both the anesthesiologist and surgeon achieve enough skills in the procedures, major operations such as lobectomies can be attempted.

The required minimum experience for thoracoscopic operations in nonintubated patients is not yet defined. However, little experience in VATS, especially in uniVATS can be considered an important risk-factor for conversion to general anesthesia. Recently, a low incidence of complications as well as low rate of conversion to general 
anesthesia in nonintubated major lung resections are being reported when carried out by well experienced VATS surgeons $(89,90)$.

It is very important to perform a careful selection of the patients scheduled for nonintubated uniVATS, especially during the learning curve of each surgeon (90) and to have an established second plan for "high-risk" patients. Conversion to intubated anesthesia is recommended early for major bleeding, significant surgical difficulties or persistent hypoxemia and tachypnea (80).

Current opinions suggest that the contraindications to this kind of procedure can differ from individuals, technical and anesthesiological factors $(92,93)$. Individual factors include various conditions such as obesity (body mass index greater than $30 \mathrm{~kg} / \mathrm{m}^{2}$ ), coagulation disorders, or a non-compliant psychological profile for awake surgery. This last point represents one of the most important hinder to nonintubated surgery and it should be known prior to the operative act. Within our nonintubated program (95), we have established a set of dedicated physicians for this purpose. Major technical factors contraindicating a nonintubated procedure are the presence of dense and extensive pleural adhesions, which is by itself a factor contraindicating any VATS surgery, or tumor larger than $6 \mathrm{~cm}$ or centrally located. Finally, anesthesiological contraindications for nonintubated surgery are hemodynamic instability, American Society of Anesthesiology score greater than II and expected difficult airways management.

After this necessary premise let's come back to the history and dates. In 2000, we presented a program to our Internal Review Board and Ethic Committee concerning investigational study of thoracic operations performed without the employ of general anesthesia and one-lung ventilation. A study group named "Awake Thoracic Surgery Research Group" was founded (95). It is, to our knowledge, the first dedicated to nonintubated thoracic surgery in the literature. During more than a decade this program achieved a consistent amount of knowledge about the surgery in awake patients and produced a great number of scientific publications $(68,69,81,84,95-105)$. In our program we initially privileged anesthesia delivered through an epidural catheter with a fully awake and collaborative patient (96-99). In this way we achieved the neurological surveillance that we consider a physiological pattern of monitoring. In our more recent nonintubated experiences we used an intercostal selective block that is simpler and quicker but equally effective in achieving thoracic analgesia $(69,102)$.

At the same time from conventional multiport VATS we progressively shifted to uniVATS with significant positive outcomes in postoperative recovery, patient acceptance and costs (105). To date, more than eight hundred successful, though prevalently minor operations, have been performed through this mixed anesthesiologic/surgical approach. Many pathological conditions were faced such as pneumothorax, emphysema, pleural infection, interstitial lung disease, malignant pleural effusion, peripheral lung nodules, mediastinal tumors and limited lung cancers (95). The number of major operations is still fairly limited with quite a significant rate of anesthesiologic or surgical conversion $(95,105)$.

In 2007, Al-Abullatief et al. (106) first described a nonintubated VATS lobectomy as an effort to decrease injuries related to general anesthesia, improving outcomes and reducing expenses. Furthermore, they showed the possibility of performing selected cases of major thoracic surgery, even thoracotomies, for lung resections with the patient awake or minimally sedated.

In 2010, Rocco et al. (85) treated pulmonary nodule in a complete ambulatory setting using only one-incision and awake technique.

The widest experience on major thoracic surgery in nonintubated patients must be attributed to the Taiwan University group. In 2011, Chen et al. reported their experience with nonintubated three-port thoracoscopic lobectomy for lung cancer (107). They noted that this approach could yield fewer postoperative complications and a shorter hospital stay compared with intubated anesthesia. They concluded that nonintubated VATS lobectomy and segmentectomy with mediastinal lymphadenectomy for early stage non-small cell lung cancer could be safely performed. In 2012, the same group highlighted in 285 consecutive patients the feasibility and safety of multi VATS resection performed under nonintubated anesthesia (108). In 2012, Tsai and Chen reported a case of bilateral lung resection with a nonintubated thoracoscopic technique (109). In 2013, Wu et al. (110) investigated the feasibility and safety of nonintubated VATS lobectomy in elderly patients for lung cancer. In 2014, Guo et al. (111) reported an analysis on feasibility and safety of segmentectomies in nonintubated patients. In 2014, Hung et al. (112) published a series of patients successfully operated by only infiltrating several intercostal spaces from $\mathrm{T} 3$ to $\mathrm{T} 8$.

In 2014, Ambrogi et al. (102) compared interstitial pulmonary biopsies under uniVATS with intercostal 
block versus three-port VATS with epidural anesthesia. The uniVATS under intercostal block proved better intraoperative and postoperative outcome.

In 2014, Gonzalez-Rivas et al. (89) published the first nonintubated single port incision $(2-5 \mathrm{~cm})$ VATS lobectomy in a 46-year old man with lung cancer in the right middle lobe. Local intercostal infiltration was used without vagal blockade or epidural anesthesia. Operative time was 80 minutes and the patient was discharged 36 hours after the operation with excellent postoperative recovery. It is evident that progresses in this field are extraordinary: the same year, Shao et al. published a nonintubated complete thoracoscopic bronchial sleeve resection for central lung cancer (113).

To date, comparative studies have successfully demonstrated that nonintubated anesthesia does not compromise the extent of lymph node dissection when vagal blockade is used to control coughing reflex (112). A number of studies have signaled benefits and limits of non-intubated VATS under locoregional anesthesia. Deng et al. in a recent meta-analysis concluded that this technique proved to be safe and feasible in the short term period (114).

Certainly, in this review we have elapsed many important contributes to history of VATS. Indeed, many other authors reported their experience with both intubated and nonintubated uniportal technique for a number of operations with good postoperative outcomes. Every year, the literature related to this topic increases dramatically, revealing a great interest for uniportal and nonintubated thoracic surgery. On the other side, an increasing number of centers all over the world prefers uniportal surgery. This is a palpable sign that many surgeons learnt and progressed in minimally invasive thoracic surgery with the paradox effect of improving the traditional open thoracic surgery as well.

In these last two decades thoracic surgery had a revolutionary change and tremendous jump forward and this was mainly due to the advent of VATS at the beginning of the '90s.

For many years, the thoracic surgeon had been anchored to the classic, traditional accesses that, with a variable width and with some combined variances, allowed a safe control of organs and structures during the procedures. However, these approaches compelled to accept several inconveniences such as acute or chronic postoperative pain, extensive and often disfiguring scars, prolonged hospitalizations, high morbidities and elevated costs. The surgeon, as a first actor, caused an indelible both physical and psychical mark that the patients recorded as the worst trauma ever felt in their life.
Now, high definition cameras, energy devices, sealing devices for vessels and small bronchi, articulated and renewed endostaplers and many other specialized and refined surgical instruments, allow surgeons to perform every procedure through $2-3$ or, once acquired adequate experience, even one single limited skin incision.

The history demonstrated that, within a five-year period, the experts in multiport VATS have been able to accomplish in uniVATS progressively more complex surgical procedures such as lung resections, bronchial sleeve, and carinal and vascular reconstructions. So, we can say in color that VATS gave birth quickly the uniVATS.

Over the years, uniVATS has increased safety and achieved as equal surgical efficacy as open surgery. uniVATS provoked a wave of enthusiasm greater than that generated by conventional VATS. However, the most surprising thing is its rapid spread, with the pattern of a real epidemic. Hundreds of scientific publications, focused issues, dedicated monographies, film series, live surgery events, experimental courses, contributed to this fast diffusion all over the world.

This new surgical modality had a fertile soil in the new surgical generations attracted by both the elevate challenge and advanced technology. Many dedicated centers were born and all these gave a contribution in pushing forward the frontiers of uniVATS that at present cannot ignore an attentive patient selections and adequate surgical formation.

The development and consolidation of conventional VATS and of uniVATS renewed and restarted the interest for operative thoracoscopy in an awake modality. The revival of nonintubated thoracic surgery paralleled the improvements and the widespread acceptance of VATS evolution. Nowadays, nonintubated anesthetic technique in thoracic interventions appears innovative and exciting. They aim at reducing the invasiveness of both multiport and uniport approach of VATS. The true benefits of nonintubated uniVATS should be further verified in future prospective studies. Further investigations are necessary to clarify the applicability and benefits of this technique for specific patient groups. However, these inseparable technological and surgical progresses have generated more confidence in the patients, even elderly, which more easily accept the proposed surgical procedure. Nowadays, combined nonintubated multiport or uniVATS allow major resections in high-risk patients with no difference in overall survival when compared with standard-risk patients.

The nonintubated uniVATS shows decreased morbidity compared with open resections. As a consequence, this 
approach should be considered for patients who historically may not have been scheduled for surgical lung resection.

From Jacobaeus's “small single-skin incision awake operation" (6) to true nonintubated uniVATS technique (115) more than one century have passed. The Jacobaeus operation survived despite its limited diffusion. Now it is as relevant as ever. The modern uniVATS story is happening in a short period of time. Its future appears incredibly open, and relentless advances like the modern naked eye 3-D image system, wireless camera and improved robotic techniques. The uniVATS history is included in a short period of time and in a succession of dates signaling its unstoppable evolution. All this continuous pathway of new conquests is due to the efforts of both single surgeons and surgical teams.

In this at historical glance of uniVATS we are pleased to tribute proper credit to the surgeon Diego GonzalezRivas from La Coruña Hospital as a true motive power in VATS evolution. He is a globally renowned expert in this novel field of thoracic surgery. His efforts tend towards different directions spending all one's lot of energy on instrumental innovations, technical solutions and constant teaching everywhere in the world. We acknowledge him the merit of having endeavored never-tried procedures with apparent courage, but based on a solid experience of traditional thoracic and experimental surgery as well. We cannot deny that he greatly contributed in the formation of a new generation of surgeons to whom he transferred his enthusiasm and competence as well as creation of new highspecialized centers.

\section{The final score}

Recently, Zhao et al. (116) wondered if nonintubated VATS can represent the final frontier. Personally, as senior thoracic surgeons who dealt with the many phases of the modern thoracic surgery, we can answer "no". Indeed, we are perfectly convinced, as now as in the past decades, that the attractive of the surgery is always alive and irresistible, because it is an art under continuous renovation. Having firmed the statement "patient safety occupies the first rank", we want to remark that the surgeon has still the task of choosing the best access and the optimal technique and the most advanced surgical procedure. All these requirements will provide the success and the progress as well. Despite the well-known values and benefits of uniVATS, the history of this technique remains open and to be written. Longer follow up and relevant prospective studies may reveal novel and multifaceted virtues and indications for uniVATS. It must stand the test of the time, which is the only that definitively establishes the validity of a surgical procedure.

\section{Acknowledgements}

We feel in debt with Dr. Gabriele Mazzitelli for his very precious and attentive cooperation in retrieving the sources of this review.

\section{Footnote}

Conflicts of Interest: The authors have no conflicts of interest to declare.

\section{References}

1. Roviaro G, Rebuffat C, Varoli F, et al. Videoendoscopic pulmonary lobectomy for cancer. Surg Laparosc Endosc 1992;2:244-7.

2. Landreneau RJ, Mack MJ, Hazelrigg SR, et al. Videoassisted thoracic surgery: basic technical concepts and intercostal approach strategies. Ann Thorac Surg 1992;54:800-7.

3. McKenna RJ Jr. Lobectomy by video-assisted thoracic surgery with mediastinal node sampling for lung cancer. J Thorac Cardiovasc Surg 1994;107:879-81.

4. Yim AP, Ho JK, Chung SS, et al. One hundred and sixtythree consecutive video thoracoscopic procedures: the Hong Kong experience. Aust N Z J Surg 1994;64:671-5.

5. McKenna RJ Jr, Houck W, Fuller CB. Video-assisted thoracic surgery lobectomy: experience with 1,100 cases. Ann Thorac Surg 2006;81:421-5.

6. Jacobaeus HC. Über die Möglichkeit die Zystoskopie bei Untersuchung seröser Höhlungen anzuwended. Munch Med Wochenschr 1910;57:2.

7. Loddenkemper R, Mathur PN, Lee P, et al. History and clinical use of thoracoscopy/pleuroscopy in respiratory medicine. Breathe 2011 8:144-55.

8. Moisiuc FV, Colt HG. Thoracoscopy: origins revisited. Respiration 2007;74:344-55.

9. Jacobaeus HC. Die Thorakoskopie und ihre praktische Bedeutung. Ergebn Ges Med 1925;7:112-66.

10. Braimbridge MV. The history of thoracoscopic surgery. Ann Thorac Surg 1993;56:610-4.

11. Prabhu VG, Narashiman R. The role of pleuroscopy in undiagnosed exudative pleural effusion. Lung India 2012;29:128-30. 
12. Thomas PA Jr. A thoracoscopic peek: what did Jacobaeus see? Ann Thorac Surg 1994;57:770-1.

13. Guido W, Gonzalez-Rivas D. Uniportal video-assisted thoracoscopic right upper sleeve lobectomy. J Vis Surg 2015;1:10.

14. Drevet G, Ugalde Figueroa P. Uniportal video-assisted thoracoscopic surgery: safety, efficacy and learning curve during the first 250 cases in Quebec, Canada. Ann Cardiothorac Surg 2016;5:100-6.

15. Yu PS, Capili F, Ng CS. Single port VATS: recent developments in Asia. J Thorac Dis 2016;8:S302-7.

16. Abu Akar F, Gonzalez-Rivas D, Ismail M, et al. Uniportal video-assisted thoracic surgery: the Middle East experience. J Thorac Dis 2017;9:871-7.

17. Ismail M, Helmig M, Swierzy M, et al. Uniportal VATS: the first German experience. J Thorac Dis 2014;6:S650-5.

18. Wu CY, Heish MJ, Wu CF. Single port VATS mediastinal tumor resection: Taiwan experience. Ann Cardiothorac Surg 2016;5:107-11.

19. Gonzalez-Rivas D. Uniportal thoracoscopic surgery: from medical thoracoscopy to non-intubated uniportal videoassisted major pulmonary resections. Ann Cardiothorac Surg 2016;5:85-91.

20. Gonzalez-Rivas D, de la Torre M, Fernandez R, et al. Singleport video-assisted thoracoscopic left upper lobectomy. Interact Cardiovasc Thorac Surg 2011;13:539-41.

21. Gonzalez-Rivas D, Fieira E, Delgado M, et al. Is uniportal thoracoscopic surgery a feasible approach for advanced stages of non-small cell lung cancer? J Thorac Dis 2014;6:641-8.

22. Yamamoto H, Okada M, Takada $M$, et al. Video-assisted thoracic surgery through a single skin incision. Arch Surg 1998;133:145-7.

23. Nesher N, Galili R, Sharony R, et al. Videothoracoscopic sympathectomy (VATS) for palmar hyperhidrosis: summary of a clinical trial and surgical results. Harefuah 2000;138:913-6.

24. Migliore M, Giuliano R, Deodato G. Video-assisted thoracic surgery through a single port. Thoracic surgery and interdisciplinary symposium on the threshold of the third millennium. An international continuing medical education programme. Naples, Italy 2000:29-30. Available online: http://xoomer.virgilio.it/naples2000/index.html

25. Migliore M, Deodato G. Single-trocar technique for minimally-invasive surgery of the chest. Surg Endosc 2001;15:899-901.

26. Migliore M. Efficacy and safety of single-trocar technique for minimally invasive surgery of the chest in the treatment of noncomplex pleural disease. J Thorac Cardiovasc Surg 2003;126:1618-23.

27. Migliore M, Calvo D, Criscione A, et al. Uniportal video assisted thoracic surgery: summary of experience, minireview and perspectives. J Thorac Dis 2015;7:E378-80.

28. Migliore M. Initial History of Uniportal Video-Assisted Thoracoscopic Surgery. Ann Thorac Surg 2016;101:412-3.

29. Rocco G, Martin-Ucar A, Passera E. Uniportal VATS wedge pulmonary resections. Ann Thorac Surg 2004;77:726-8.

30. Rocco G. History and indications of uniportal pulmonary wedge resections. J Thorac Dis 2013;5 Suppl 3:S212-3.

31. Rocco G, Martucci N, La Manna C, et al. Ten-year experience on 644 patients undergoing single-port (uniportal) video-assisted thoracoscopic surgery. Ann Thorac Surg 2013;96:434-8.

32. Jutley RS, Khalil MW, Rocco G. Uniportal vs standard three-port VATS technique for spontaneous pneumothorax: comparison of post-operative pain and residual paraesthesia. Eur J Cardiothorac Surg 2005;28:43-6.

33. Reinersman JM, Passera E, Rocco G. Overview of uniportal video-assisted thoracic surgery (VATS):past and present. Ann Cardiothorac Surg 2016;5:112-7.

34. Gonzalez D, Delgado M, Paradela M, et al. Uni-incisional video-thoracoscopic left lower lobectomy in an incomplete fissure. Innovations (Phila) 2011;6:45-7.

35. Gonzalez-Rivas D, Fieira E, Mendez L, et al. Single-port video-assisted thoracoscopic anatomic segmentectomy and right upper lobectomy. Eur J Cardiothorac Surg $2012 ; 42:$ e169-71.

36. Gonzalez-Rivas D, de la Torre M, Fernandez R, et al. Video: Single-incision video-assisted thoracoscopic right pneumonectomy. Surg Endosc 2012;26:2078-9.

37. Gonzalez-Rivas D, Fernandez R, Fieira E, et al. Singleincision thoracoscopic right upper lobectomy with chest wall resection by posterior approach. Innovations (Phila) 2013;8:70-2.

38. Gonzalez-Rivas D, Fernandez R, Fiera E, et al. Uniportal video-assisted thoracoscopic bronchial sleeve lobectomy: first report. J Thorac Cardiovasc Surg 2013;145:1676-7.

39. Gonzalez-Rivas D, Delgado M, Fieira F, et al. Single-port video-assisted thoracoscopic lobectomy with pulmonary artery reconstruction. Interact Cardiovasc Thorac Surg 2013;17:889-91.

40. Gonzalez-Rivas D, Paradela M, Fernandez R, et al. Uniportal video-assisted thoracoscopic lobectomy: two years of experience. Ann Thorac Surg 2013;95:426-32.

41. Gonzalez-Rivas D, Delgado M, Fieira F, et al. Left lower 
sleeve lobectomy by uniportal video-assisted thoracoscopic approach. Interact Cardiovasc Thorac Surg 2014;18:237-9.

42. Gonzalez-Rivas D, Fieira E, de la Torre M, et al. Bronchovascular right upper lobe reconstruction by uniportal video-assisted thoracoscopic surgery. J Thorac Dis 2014;6:861-3.

43. Gonzalez-Rivas D, Delgado M, Fieria E. Double sleeve uniportal video-assisted thoracoscopic lobectomy for nonsmall lung cancer. Ann Cardiothorac Surg 2014;3:E2.

44. Gonzalez-Rivas D, Fieira E, Delgado M, et al. Uniportal video-assisted thoracoscopic sleeve lobectomy and other complex resections. J Thorac Dis 2014;6:S674-81.

45. Gonzalez-Rivas D, Xie B, Yang Y, et al. Uniportal videoassisted thoracoscopic lobectomy with en-bloc chest wall resection. J Vis Surg 2015;1:7.

46. Gonzalez-Rivas D, Yang Y, Stupnik T, et al. Uniportal video-assisted thoracoscopic bronchovascular, tracheal and carinal sleeve resections. Eur J Cardiothorac Surg 2016;49 Suppl 1:i6-16.

47. Zhang W, Wei Y, Jiang H, et al. Thoracotomy is better than thoracoscopic lobectomy in the lymph node dissection of lung cancer: a systematic review and metaanalysis. World J Surg Oncol 2016;14:290.

48. Delgado Roel M, Roel M, Fieira Costa EM, et al. Uniportal video-assisted thoracoscopic lymph node dissection. J Thorac Dis 2014;6:S665-8.

49. Liu CC, Shah CS, Pennarun N, et al. Transition from a multiport technique to a single-port technique for lung cancer surgery: is lymph node dissection inferior using the single-port technique? Eur J Cardiothorac Surg 2016;49:i64-72.

50. Mu JW, Gao SG, Xue Q, et al. A matched comparison study of uniportal versus triportal thoracoscopic lobectomy and sublobectomy for early-stage nonsmall cell lung cancer. Chin Med J (Engl) 2015;128:2731-5.

51. Shen G, Chai Y, Huang L, et al. Uniportal video-assisted thoracoscopic right upper lobectomy with systematic lymphadenectomy. J Thorac Dis 2016;8:2275-80.

52. Guido Guerrero W, Gonzalez-Rivas D, Hernandez Arenas LA, et al. Techniques and difficulties dealing with hilar and interlobar benign lymphadenopathy in uniportal VATS. J Vis Surg 2016;2:23.

53. Gonzalez D, de la Torre M, Paradela M, et al. Videoassisted thoracic surgery lobectomy:3-year initial experience with 200 cases. Eur J Cardiothorac Surg 2011;40:e21-8.

54. Desai H, Natt B, Kim S, et al. Decreased in-hospital mortality after lobectomy using video-assisted thoracoscopic surgery compared with open thoracotomy. Ann Am Thorac Soc 2017;14:262-6.

55. Agostini P, Lugg ST, Adams K, et al. Postoperative pulmonary complications and rehabilitation requirements following lobectomy: a propensity score matched study of patients undergoing video-assisted thoracoscopic surgery versus thoracotomy. Interact Cardiovasc Thorac Surg 2017;24:931-7.

56. Bendixen M, Jørgensen OD, Kronborg C, et al. Postoperative pain and quality of life after lobectomy via video-assisted thoracoscopic surgery or anterolateral thoracotomy for early stage lung cancer: a randomised controlled trial. Lancet Oncol 2016;17:836-44.

57. Qiang G, Liang C, Guo Y, et al. Video-assisted thoracoscopic lobectomy for elderly nonsmall cell lung cancer: Short-term and long-term outcomes. J Cancer Res Ther 2015;11:793-7.

58. Jawitz OK, Wang Z, Boffa DJ, et al. The differential impact of preoperative comorbidity on perioperative outcomes following thoracoscopic and open lobectomies. Eur J Cardiothorac Surg 2017;51:169-74.

59. Tamura M, Shimizu Y, Hashizume Y. Pain following thoracoscopic surgery: retrospective analysis between single-incision and three-port video-assisted thoracoscopic surgery. J Cardiothorac Surg 2013;8:153.

60. McElnay PJ, Molyneux M, Krishnadas R, et al. Pain and recovery are comparable after either uniportal or multiport video-assisted thoracoscopic lobectomy: on observation study. Eur J Cardiothorac Surg 2015;47:912-5.

61. Harris CG, James RS, Tian DH, et al. Systematic review and meta-analysis of uniportal versus multiportal videoassisted thoracoscopic lobectomy for lung cancer. Ann Cardiothorac Surg 2016;5:76-84.

62. Dai F, Meng S, Mei L, et al. Single-port video-assisted thoracic surgery in the treatment of non-small cell lung cancer: a propensity-matched comparative analysis. J Thorac Dis 2016;8:2872-8.

63. French DG, Thompson C, Gilbert S. Transition from multiple port to single port video-assisted thoracoscopic anatomic pulmonary resection: early experience and comparison of perioperative outcomes. Ann Cardiothorac Surg 2016;5:92-9.

64. Chang JM, Kam KH, Yen YT, et al. From biportal to uniportal video-assisted thoracoscopic anatomical lung resection: a single-institute experience. Medicine (Baltimore) 2016;95:e5097.

65. Nagahiro I, Andou A, Aoe M, et al. Pulmonary function, postoperative pain, and serum cytokine level after 
lobectomy: a comparison of VATS and conventional procedure. Ann Thorac Surg 2001;72:362-5.

66. Vanni G, Tacconi F, Sellitri F, et al. Impact of awake videothoracoscopic surgery on postoperative lymphocyte responses. Ann Thorac Surg 2010;90:973-8.

67. Zhang LB, Wang B, Wang XY, et al. Influence of videoassisted thoracoscopic lobectomy on immunological functions in non-small cell lung cancer patients. Med Oncol 2015;32:201.

68. Mineo TC, Sellitri F, Vanni G, et al. Immunological and inflammatory impact of non-intubated lung metastasectomy. Int J Mol Sci 2017;18. pii:E1466.

69. Ambrogi V, Sellitri F, Perroni G, et al. Uniportal videoassisted thoracic surgery colorectal lung metastasectomy in non-intubated anesthesia. J Thorac Dis 2017;9:254-61.

70. Gonzalez-Rivas D, Stupnik T, Fernandez R, et al. Intraoperative bleeding control by uniportal video-assisted thoracoscopic surgery. Eur J Cardiothorac Surg 2016;49 Suppl 1:117-24.

71. Sihoe AD. The evolution of minimally invasive thoracic surgery:implications for the practice of uniportal thoracoscopic surgery. J Thorac Dis 2014;6:S604-17.

72. Ng CS, Kim HK, Wong RH, et al. Single-port videoassisted thoracoscopic major lung resections: experience with 150 consecutive cases. Thorac Cardiovasc Surg 2016;64:348-53.

73. Liu CC, Wang BY, Shih CS, et al. Subxiphoid singleincision thoracoscopic left upper lobectomy. J Thorac Cardiovasc Surg 2014;148:3250-1.

74. Liu CC, Shih CS, Liu YH, et al. Subxiphoid singleport video-assisted thoracoscopic surgery. J Vis Surg 2016;2:112.

75. Yano M, Moriyama S, Haneda H, et al. The subxiphoid approach leads to less invasive thoracoscopic thymectomy than the lateral approach. World J Surg 2017;41:763-70.

76. Kido T, Hazama K, Inoue Y, et al. Resection of anterior mediastinal masses through an infrasternal approach. Ann Thorac Surg 1999;67:263-5.

77. Suda T. Uniportal subxiphoid video-assisted thoracoscopic thymectomy. J Vis Surg 2016;2:123.

78. Suda T. Single port thymectomy using a subxiphoid approach-surgical technique. Ann Cardiothorac Surg 2016;5:56-8.

79. Chen H, Xu G, Zheng W, et al. Video-assisted thoracoscopic extended thymectomy using the subxiphoid approach. J Vis Surg 2016;2:157.

80. Irons JF, Martinez G. Anaesthetic considerations for nonintubated thoracic surgery. J Vis Surg 2016;2:61.
81. Mineo TC, Tacconi F. From "awake" to "monitored anesthesia care" thoracic surgery: a 15-year evolution. Thorac Cancer 2014;5:1-13.

82. Gonzalez-Rivas D, Bonome C, Fieira E, et al. Nonintubated video-assisted thoracoscopic lung resections: the future of thoracic surgery? Eur J Cardiothorac Surg 2016;49:721-31.

83. Sugasawa Y, Yamaguchi K, Kumakura S, et al. The effect of one-lung ventilation upon pulmonary inflammatory responses during lung resection. J Anesth 2011;25:170-7.

84. Tacconi F, Pompeo E, Sellitri F, et al. Surgical stress hormones response is reduced after awake videothoracoscopy. Interact Cardiovasc Thorac Surg 2010;10:666-71.

85. Rocco G, Romano V, Accardo R, et al. Awake singleaccess (uniportal) video-assisted thoracoscopic surgery for peripheral pulmonary nodules in a complete ambulatory setting. Ann Thorac Surg 2010;89:1625-7.

86. Ng CS, Gonzalez-Rivas D, D'Amico TA, et al. Uniportal VATS-a new era in lung cancer surgery. J Thorac Dis 2015;7:1489-91.

87. Liu YJ, Hung MH, Hsu HH, et al. Effects on respiration of nonintubated anesthesia in thoracoscopic surgery under spontaneous ventilation. Ann Transl Med 2015;3:107.

88. Irons JF, Miles LF, Joshi KR, et al. Intubated versus nonintubated general anesthesia for video-assisted thoracoscopic surgery-a case-control study. J Cardiothorac Vasc Anesth 2017;31:411-7.

89. Gonzalez-Rivas D, Fernandez R, de la Torre M, et al. Single-port thoracoscopic lobectomy in a nonintubated patient: the least invasive procedure for a major resection? Interact Cardiovasc Thorac Surg 2014;19:552-5.

90. Gonzalez-Rivas D, Aymerich H, Bonome C, et al. From open operations to non-intubated uniportal video-assisted thoracoscopic lobectomy: minimizing the trauma to the patient. Ann Thorac Surg 2015;100:2003-5.

91. Gonzalez-Rivas D, de la Torre M, Fernandez R, et al. Uniportal video-assisted thoracoscopic left upper lobectomy under spontaneous ventilation. J Thorac Dis 2015;7:494-5.

92. Hung WT, Hsu HH, Hung MH, et al. Nonintubated uniportal thoracoscopic surgery for resection of lung lesions. J Thorac Dis 2016;8:S242-50.

93. Yang JT, Hung MH, Chen JS, et al. Anesthetic considerations for nonintubated VATS. J Thorac Dis 2014;6:10-3.

94. Kiss G, Castillo M. Nonintubated anesthesia in thoracic surgery: general issues. Ann Transl Med 2015;3:110. 
95. Mineo TC, Tamburrini A, Perroni G, et al. 1000 cases of tubeless video-assisted thoracic surgery at the Rome Tor Vergata University. Future Oncol 2016;12:13-8.

96. Pompeo E, Mineo D, Rogliani P, et al. Feasibility and results of awake thoracoscopic resection of solitary pulmonary nodules. Ann Thorac Surg 2004;78:1761-8.

97. Mineo TC, Pompeo E, Mineo D, et al. Awake nonresectional lung volume reduction surgery. Ann Surg 2006;243:131-6.

98. Pompeo E, Tacconi F, Mineo TC. Awake video-assisted thoracoscopic biopsy in complex anterior mediastinal masses. Thorac Surg Clin 2010;20:225-33.

99. Tacconi F, Pompeo E, Fabbi E, et al. Awake videoassisted pleural decortication for empyema thoracis. Eur J Cardiothorac Surg 2010;37:594-601.

100. Mineo TC, Ambrogi V. Efficacy of awake thoracic surgery. J Thorac Cardiovasc Surg 2012;143:249-50.

101. Mineo TC, Ambrogi V. Awake thoracic surgery for secondary spontaneous pneumothorax: another advancement. J Thorac Cardiovasc Surg 2012;144:1533-4.

102. Ambrogi V, Mineo TC. VATS biopsy for undetermined interstitial lung disease under non-general anesthesia:comparison between uniportal approach under intercostal block vs. three-ports in epidural anesthesia. J Thorac Dis 2014;6:888-95.

103. Mineo TC, Tacconi F, Ambrogi V, et al. Nonintubated VATS segmentectomy: when and for whom? Ann Thorac Surg 2014;98:388.

104. Mineo TC, Sellitri F, Tacconi F, et al. Quality of life and outcomes after nonintubated versus intubated videothoracoscopic pleurodesis for malignant pleural effusion: comparison by a case-matched study. J Palliat Med 2014;17:761-8.

105. Mineo TC, Ambrogi V, Sellitri F. Non-intubated videoassisted thoracic surgery from multi to uniport approaches. Single centre experience. EMJ Respir 2016;4:104-12.

106.Al-Abdullatief M, Wahood A, Al-Shirawi, et al. Awake

doi: 10.21037/jovs.2017.10.11

Cite this article as: Mineo TC, Ambrogi V. A glance at the history of uniportal video-assisted thoracic surgery. J Vis Surg 2017;3:157. anesthesia for major thoracic surgical procedures: an observational study. Eur J Cardiothorac Surg 2007;32:346-50.

107. Chen JS, Cheng YJ, Hung MH, et al. Nonintubated thoracoscopic lobectomy for lung cancer. Ann Surg 2011;254:1038-43.

108. Chen KC, Cheng YJ, Hung MH, et al. Nonintubated thoracoscopic lung resection: a 3-year experience with 285 cases in a single institution. J Thorac Dis 2012;4:347-51.

109. Tsai TM, Chen JS. Nonintubated thoracoscopic surgery for pulmonary lesions in both lungs. J Thorac Cardiovasc Surg 2012;144:e95-7.

110.Wu CY, Chen JS, Lin YS, et al. Feasibility and safety of nonintubated thoracoscopic lobectomy for geriatric lung cancer patients. Ann Thorac Surg 2013;95:405-11.

111. Guo Z, Shao W, Yin W, et al. Analysis of feasibility and safety of complete video-assisted thoracoscopic resection of anatomic pulmonary segments under non-intubated anesthesia. J Thorac Dis 2014;6:37-44.

112.Hung MH, Hsu HH, Chan KC, et al. Non-intubated thoracoscopic surgery using internal intercostal nerve block, vagal block and targeted sedation. Eur J Cardiothorac Surg 2014;46:620-5.

113. Shao W, Phan K, Guo X, et al. Non-intubated complete thoracoscopic bronchial sleeve resection for central lung cancer. J Thorac Dis 2014;6:1485-8.

114. Deng HY, Zhu ZJ, Wang YC, et al. Non-intubated video-assisted thoracoscopic surgery under loco-regional anaesthesia for thoracic surgery: a meta-analysis. Interact Cardiovasc Thorac Surg 2016;23:31-40.

115. Migliore M, Giuliano R, Aziz T, et al. Four-step local anesthesia and sedation for thoracoscopic diagnosis and management of pleural disease Chest 2002;121:2032-5.

116.Zhao ZR, Lau RW, Ng CS. Non-intubated video-assisted thoracic surgery: the final frontier? Eur J Cardiothorac Surg 2016;50:925-6. 